\title{
Colonic M1 macrophage is associated with the prolongation of gastrointestinal motility and obesity in mice treated with vancomycin
}

\author{
YOSHIHITO INOUE ${ }^{1}$, HIROKAZU FUKUI ${ }^{1}$, XIN XU $^{1,2}$, YING RAN $^{1,2}$, TOSHIHIKO TOMITA $^{1}$, \\ TADAYUKI OSHIMA $^{1}$, JIRO WATARI ${ }^{1}$ and HIROTO MIWA ${ }^{1}$ \\ ${ }^{1}$ Division of Gastroenterology, Department of Internal Medicine, Hyogo College of Medicine, Nishinomiya, \\ Hyogo 663-8501, Japan; ${ }^{2}$ Department of Gastroenterology and Hepatology, \\ Tianjin Medical University General Hospital, Tianjin 300052, P.R. China
}

Received October 22, 2018; Accepted January 28, 2019

DOI: $10.3892 / \mathrm{mmr} .2019 .9920$

\begin{abstract}
Gut microbiota plays a pivotal role in not only the gastrointestinal (GI) immune system but also GI motility and metabolism. Antibiotic treatments are likely to affect the gut flora and GI immune system, subsequently disturbing GI motility and body metabolism. In the present study, we investigated antibiotic-induced alterations of body metabolism and GI motility in association with the macrophage profile in the colon. Specific pathogen-free (SPF) mice (ICR; 6 weeks old; female) were orally administered vancomycin $(0.2 \mathrm{mg} / \mathrm{ml})$ in drinking water for 5 weeks, and subsequent changes in pathophysiology were observed. The expression of CD80 and CD163 was examined by immunohistochemistry and the expression of cytokines in colonic tissues was evaluated by reverse transcription-quantitative polymerase chain reaction. The gastrointestinal transit time (GITT) was measured by administration of carmine red $(6 \% \mathrm{w} / \mathrm{v})$ solution. In the vancomycin-treated SPF mice, significant increases in body weight, cecum weight and GITT were observed compared with the controls. The number of CD80-positive M1 macrophages and the expression of interferon- $\gamma$ and interleukin-12 were significantly increased, whereas, the numbers of CD163-positive M2 macrophages in the mucosal and muscular layers were decreased in the colon of vancomycin-treated mice. GITT was positively correlated with the number of CD80-positive M1 macrophages in the colonic mucosa;
\end{abstract}

Correspondence to: Dr Hirokazu Fukui, Division of Gastroenterology, Department of Internal Medicine, Hyogo College of Medicine, 1-1 Mukogawa, Nishinomiya, Hyogo 663-8501, Japan E-mail: hfukui@hyo-med.ac.jp

Abbreviations: GI, gastrointestinal; GITT, gastrointestinal transit time

Key words: macrophage, polarization, CD80, CD163, gastrointestinal motility, microbiota, cytokines however, was negatively correlated with the number of CD163-positive M2 macrophages in the mucosal and muscular layers. Therefore, it was suggested that antibiotic treatment affects body metabolism and GI motility, accompanied by alterations in macrophage polarization and cytokine profiles in the colon.

\section{Introduction}

Gut microbiota play a pivotal role in various aspects of host physiology including the immune system, metabolism, hormonal secretion and gastrointestinal (GI) motility (1). The mechanism by which gut microbiota affects such physiological functions remains largely unclear. However, immune cells appear to be key players in mediating the interaction between gut microbiota and host tissues. Among various immune cells, macrophages have been highlighted due to their polarization into different phenotypes, M1 and M2, whose profiles are closely associated with specific cytokines $(2,3)$. Indeed, the T helper cell type 1 (Th1) signature cytokine, interferon- $\gamma($ IFN- $\gamma)$, is associated with M1 polarization towards a proinflammatory phenotype (4), whereas macrophages exposed to Th2 signature cytokines, such as IL-4, assume an anti-inflammatory phenotype referred to as M2 polarization (5). M1 and M2 macrophages produce mainly inflammatory and anti-inflammatory cytokines, respectively (6). Furthermore, M1 and M2 macrophages have been reported to share dominancy of their roles, including antigen presentation, phagocytosis, and growth factor/cytokine secretion to enteric neurons (7).

Antibiotic treatment is a critical factor causing imbalance of the gut microbiota profile (dysbiosis). Antibiotics are dispensable for the treatment of infection, but the dysbiosis they cause leads to not only Clostridium difficile colitis (8) but also adiposity, insulin resistance or functional gastrointestinal disorders (9). In particular, it is noteworthy that dysbiosis occurring in the early stage of life may be a significant factor in the development of disorders of metabolism and gastrointestinal motility (10). In this context, we prepared an animal model subjected to treatment with the antibiotic vancomycin 
that has been widely used and its related data is well accumulated. Thereafter, we investigated the resulting alterations of body metabolism and gastrointestinal motility in relation to the macrophage profile in the colon.

\section{Materials and methods}

Antibiotic treatment. Specific pathogen-free (SPF) mice (ICR, 6 weeks old, female) were obtained from Clea Japan (Tokyo, Japan). To create dysbiotic conditions for gut microbiota, the SPF mice were orally administered vancomycin $(0.2 \mathrm{mg} / \mathrm{ml}$; Sigma, St. Louis, MO, USA) in drinking water for five weeks, whereas controls were supplied with untreated water (11). Body weight and 24-h food intake were monitored weekly. At the end point of the experiments, the mice were fasted for $4 \mathrm{~h}$ before sacrifice. The length of the small intestine and colon, and the weight of the cecal content, were measured. The GI tissues were removed from the mice, cut open along the longitudinal axis, rinsed with saline, and fixed in neutral aqueous phosphate-buffered $10 \%$ formalin for histological examination or stored in nitrogen liquid for RT-qPCR. The experimental protocol was approved by the Animal Use and Care Committee at Hyogo College of Medicine.

Reverse transcription-quantitative polymerase chain reaction (RT-qPCR). Total RNA was isolated from the colonic tissues with TRIzol reagent (Invitrogen; Thermo Fisher Scientific, Inc., Waltham, MA, USA). Total RNA $(4 \mu \mathrm{g})$ was reverse-transcribed using oligo(dT) primer (Applied Biosystems, Branchburg, NJ, USA), and RT-qPCR was performed using a 7900H Fast Real-Time PCR System (Applied Biosystems) as described previously (12). The set of primers for mouse $I L-4$, $I L-6, I L-10, I L-12, I F N-\gamma$ and glyceraldehydes-3-phosphate dehydrogenase $(G A P D H)$ were prepared as shown in Table I. RT-qPCR assays were carried out with $200 \mathrm{ng}$ of RNA equivalent cDNA, SYBR-Green Master Mix (Applied Biosystems), and $500 \mathrm{nmol} / 1$ gene-specific primers. The PCR cycling conditions were $50^{\circ} \mathrm{C}$ for $15 \mathrm{sec}$ and $60^{\circ} \mathrm{C}$ for $60 \mathrm{sec}$. The intensity of the fluorescent dye was determined, and the expression levels of target gene mRNA were normalized to the expression level of GAPDH mRNA.

Immunohistochemistry. Immunohistochemical staining for CD80 (a marker of M1 polarized macrophages) and CD163 (a marker of M2 polarized macrophages) was performed with an Envision Kit (Dako, Kyoto, Japan) in accordance with the manufacturer's protocol (13). Polyclonal anti-CD80 antibody $(1: 10,000)$ and polyclonal anti-CD163 antibody $(1: 1,000)$ (both from Abcam, Cambridge, UK) were used as the primary antibodies. In brief, the sections were treated by microwave heating for $20 \mathrm{~min}$ in 1 Dako REAL Target Retrieval Solution (Dako Denmark, Glostrup, Denmark), preincubated with $0.3 \% \mathrm{H}_{2} \mathrm{O}_{2}$ in methanol for $20 \mathrm{~min}$ at room temperature, and incubated with the primary antibodies for $60 \mathrm{~min}$ at room temperature. The slides were then washed in PBS, incubated with a secondary antibody for $30 \mathrm{~min}$, visualized by 3,3'-diaminobenzidine tetrahydrochloride with $0.05 \% \mathrm{H}_{2} \mathrm{O}_{2}$ for $3 \mathrm{~min}$, and then counterstained with Mayer's hematoxylin. The numbers of CD80-positive and CD163-positive cells were evaluated as follows: Four sections in each mouse were
Table I. Primers for reverse transcription-polymerase chain reaction analysis.

\begin{tabular}{lll}
\hline Genes & Direction & \multicolumn{1}{c}{ Sequences } \\
\hline$I L-4$ & Forward & 5'-GAATGTACCAGGAGCGATATC-3' \\
& Reverse & 5'-CTCAGTACTACGAGTAATCCA-3' \\
$I L-6$ & Forward & 5'-CCAGTTGCCTTCTTGGGACT-3' \\
& Reverse & 5'-GGTCTGTTGGGAGTGGTATCC-3' \\
$I L-10$ & Forward & 5'-TGGACAACATACTGCTAACCG-3' \\
& Reverse & 5'-GGATCATTTCCGATAAGGCT-3' \\
$I L-12$ & Forward & 5'-CAACATCAAGAGCAGTAGCAG-3' \\
& Reverse & 5'-TACTCCCAGCTGACCTCCAC-3' \\
$I F N-\gamma$ & Forward & 5'-GCATCTTGGCTTTGCAGCT-3' \\
& Reverse & 5'-CCTTTTTCGCCTTGCTGTTG-3' \\
GAPDH & Forward & 5'-GGAGAAACCTGCCAAGTATG-3' \\
& Reverse & 5'-TGGGAGTTGCTGTTGAAGTC-3'
\end{tabular}

IL, interleukin; IFN- $\gamma$, interferon- $\gamma$.

prepared for the colon. The positive cells were counted in the lamina propria and muscular layer in at least five different visual fields in a 1,000- $\mu \mathrm{m}$ stretch of the entire length using well-oriented tissue sections, and the average was calculated for each mouse.

GI transit time (GITT). GITT was measured as described previously (14). In brief, the mice were orally treated with $0.3 \mathrm{ml}$ of $0.5 \%$ methylcellulose solution including $6 \%$ carmine red (Wako Pure Chemical Industries, Ltd., Osaka, Japan). They were then allowed access to food and water ad libitum until the first red fecal pellet appeared. GITT was determined as the time period between oral gavage and the appearance of the first red fecal pellet.

Statistical analysis. All values were expressed as the mean \pm standard error of the mean. Significance of differences between the two animal groups was analyzed by Mann-Whitney U test. Correlations among GITT, CD80 expression and CD163 expression were assessed by linear regression analysis. $\mathrm{P}<0.05$ was considered to indicate a statistically significant difference.

\section{Results}

Effect of vancomycin treatment on body weight, food intake, gastrointestinal motility and colonic morphology in mice. Body weight increased according to body growth in both the control and the vancomycin-treated groups. The percentage increase in body weight was significantly greater in vancomycin-treated mice from 2 weeks after the start of the experiment (Fig. 1A). Regarding the morphology of the colon, its length was significantly longer in vancomycin-treated mice than in controls (Fig. 1B). Additionally, we detected an apparent enlargement of the cecum in vancomycin-treated mice relative to the controls (Fig. 1C). Compatible with this 


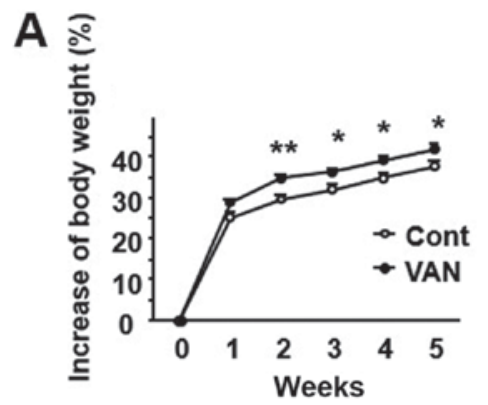

C
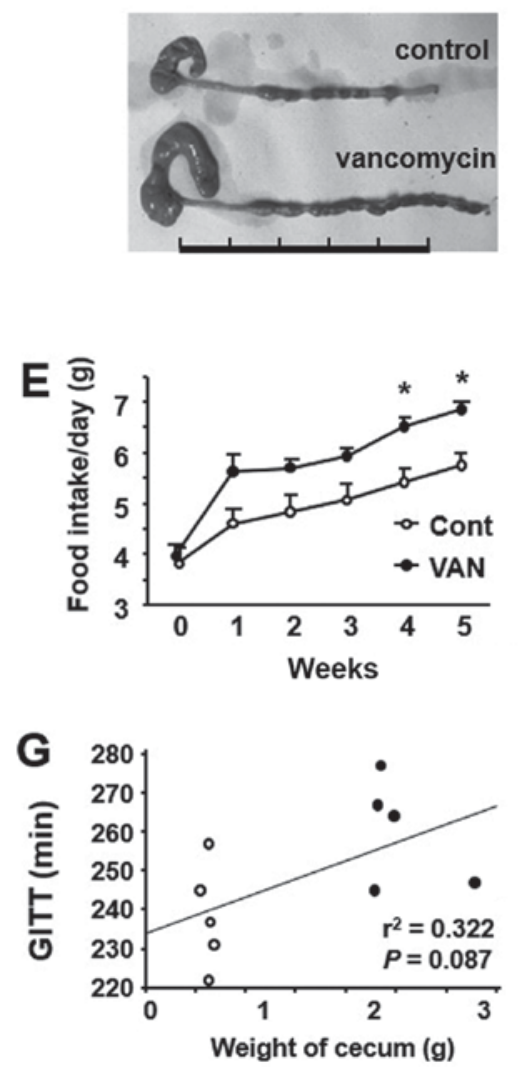

B
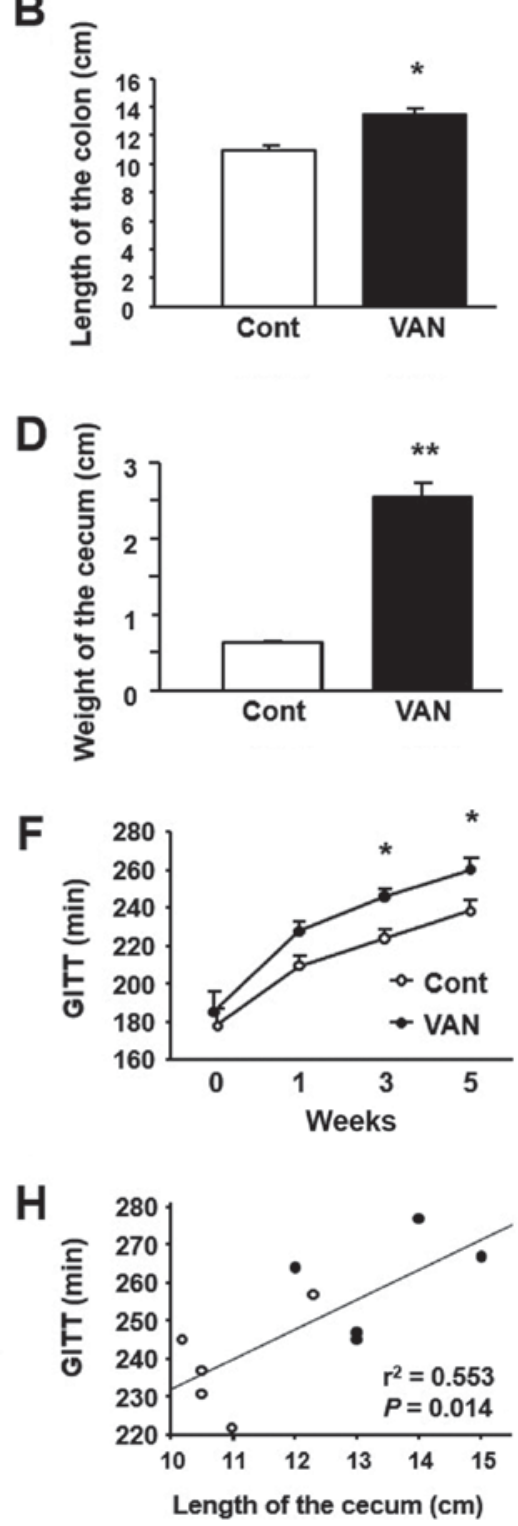

Figure 1. Effect of vancomycin treatment on body weight, food intake, gastrointestinal motility and colonic morphology in mice. (A) Change in body weight. (B) Length of colon at 5 weeks after vancomycin treatment. (C) Representative appearance of the cecum in the experimental mice treated with vancomycin. (D) Weight of cecum at 5 weeks after vancomycin treatment. (E) Change in the amount of food intake. (F) Change in GITT. Correlation between GITT and $(\mathrm{G})$ the cecum weight and $(\mathrm{H})$ the colon length. White circle, control; black circle, vancomycin group. Scale bar, $10 \mathrm{~cm}$. Results are expressed as the mean \pm standard error. $\mathrm{n}=5$ /group. ${ }^{*} \mathrm{P}<0.05,{ }^{* *} \mathrm{P}<0.01$ vs. respective Cont. VAN, vancomycin; Cont, control; GITT, gastrointestinal transit time.

finding, the weight of the cecum was significantly greater in the vancomycin-treated mice (Fig. 1D). We also investigated food intake and gastrointestinal motility in the two groups. As shown in Fig. 1E, vancomycin-treated mice showed a significant increase of food intake relative to the controls at 2 weeks or later from the start of the experiment. Furthermore, we observed that GITT was significantly prolonged in vancomycin-treated mice than in the controls beyond 4 weeks after the start of the treatment (Fig. 1F). Regarding the relationship between GITT and intestinal morphology, GITT tended to correlate to the weight of cecum (Fig. 1G) and significantly correlated with the length of colon (Fig. 1H).

Expression of CD80 in the colon of mice treated with vancomycin. CD80 expression, as a marker of M1 macrophages, was investigated in the colonic tissues by immunohistochemistry.
Immunoreactivity for CD80 was detected in immune cells in the lamina propria of the colonic mucosa (Fig. 2A) but not in the muscular layer in either control or vancomycin treated mice (data not shown). In control mice, the CD80-positive macrophages were localized mainly at the bottom of the mucosal layer. On the other hand, in vancomycin-treated mice, CD80-positive macrophages were distributed not only at bottom but also the upper part of the colonic mucosal layer (Fig. 2A). The number of CD80-positive macrophages was significantly greater in both the proximal and distal colon in vancomycin-treated mice relative to the controls (Fig. 2B).

Expression of CD163 in the colon of mice treated with vancomycin. CD163 expression, as a marker of M2 macrophages, was also investigated in the colonic tissues by immunohistochemistry. Immunoreactivity for CD163 was detected in immune 

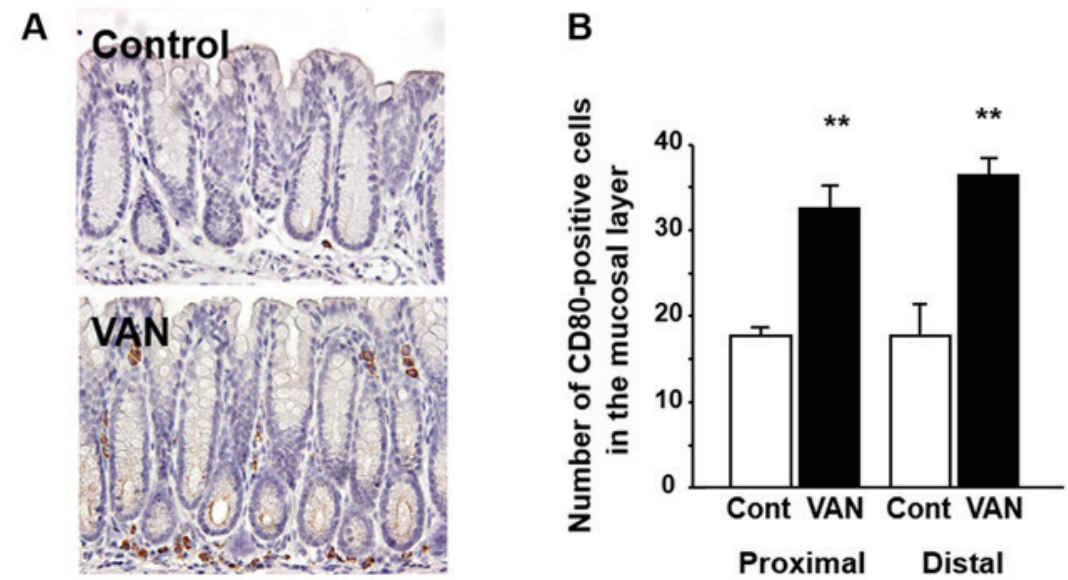

Figure 2. Effect of vancomycin treatment on CD80 expression in the colon of mice. (A) Representative immunostaining of CD80 in the colon of untreated controls and mice treated with vancomycin for 5 weeks. CD80-positive macrophages are observed in the lamina propria in the colonic mucosa. (B) Number of CD80-positive macrophages in the colon of untreated controls and mice treated with vancomycin for 5 weeks. Magnification, $\mathrm{x} 400$. All the results are expressed as the mean \pm standard error. $\mathrm{n}=5 /$ group. ${ }^{* *} \mathrm{P}<0.01$ vs. Cont. VAN, vancomycin; Cont, control.

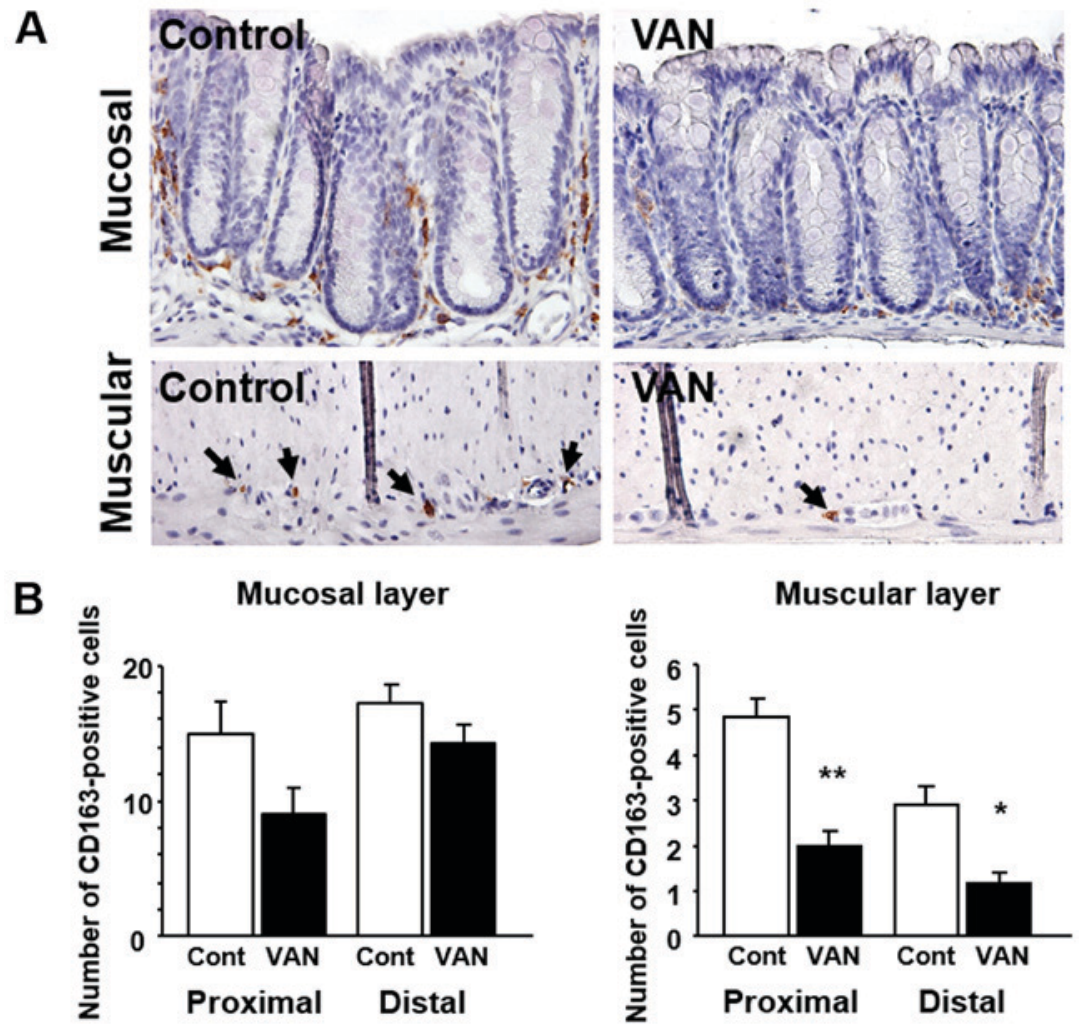

Figure 3. Effect of vancomycin treatment on CD163 expression in the colon of mice. (A) Representative immunostaining of CD163 in the colonic mucosa and muscular layer of untreated controls and mice treated with vancomycin for 5 weeks. Arrows indicate CD163-positive macrophages in the muscular layer. (B) Number of CD163-positive macrophages in the colon of untreated controls and mice treated with vancomycin for 5 weeks. Magnification, x400. All the results are expressed as the mean \pm standard error. $n=5$ /group). ${ }^{*} \mathrm{P}<0.05,{ }^{* * *} \mathrm{P}<0.01$ vs. Cont. VAN, vancomycin; Cont, control.

cells in the lamina propria of the colonic mucosa (Fig. 3A). Although their number was small, CD163-positive macrophages were detected in the muscular layer of the colon (Fig. 3A). The number of CD163-positive cells tended to be greater in both the proximal and distal colonic mucosa in vancomycin-treated mice ( $\mathrm{P}=0.075$ and 0.251 , respectively) (Fig. 3B). In the muscular layer, the number of CD163-positive cells was significantly increased in both the proximal and distal colonic mucosa in vancomycin-treated mice (Fig. 3B).
Expression of cytokines in the colon of mice treated with vancomycin. We next examined the expression of cytokines in the colonic tissue of vancomycin-treated mice. Although the expression of several cytokines differed between the controls and vancomycin-treated mice, marked differences were detected in the expression of IL-4, IL-12 and IFN- $\gamma$. In the colon of vancomycin-treated mice, IL-4 expression was significantly decreased whereas expression of IL-12 and IFN- $\gamma$ was strongly increased (Fig. 4). 

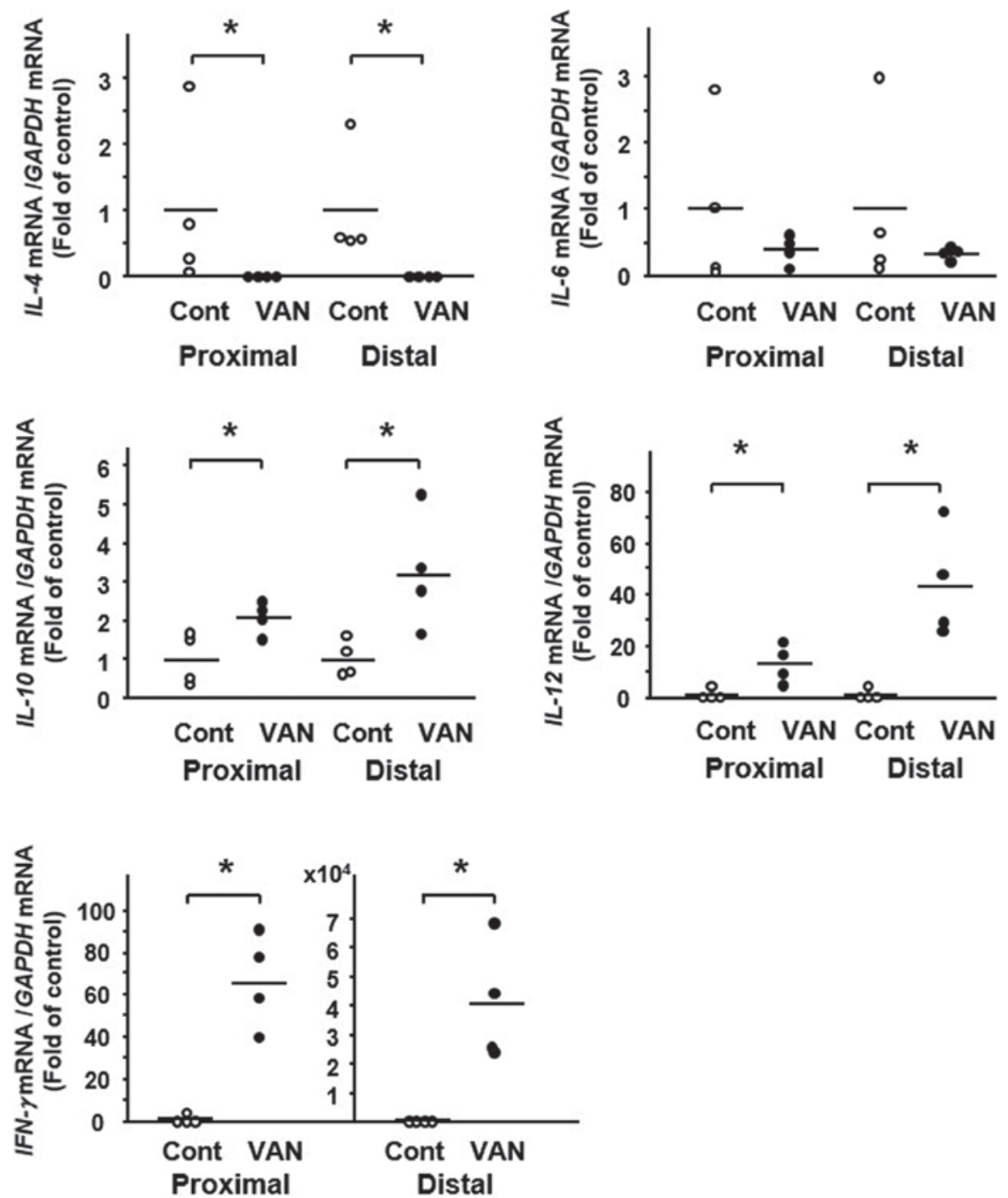

Figure 4. Effect of vancomycin treatment on cytokines expression in the colon of mice. Reverse transcription-quantitative polymerase chain reaction analysis showing a comparison of cytokine mRNA expression in colonic tissues. Results are presented for four mice per group, with bars indicating mean values. ${ }^{*} \mathrm{P}<0.05$ vs. Cont. VAN, vancomycin; Cont, control; IL, interleukin; IFN- $\gamma$, interferon- $\gamma$.

Correlation between GITT and CD80 or CD163 expression in the colon of mice. To investigate the effect of macrophage phenotype alteration on GI motility, we analyzed the correlation between GITT and CD80 or CD163 expression in the experimental mice by linear regression analysis. GITT was positively correlated with the number of CD80-positive cells in the colonic mucosa (Fig. 5A). On the other hand, GITT was negatively correlated with the number of CD163-positive cells in the colonic mucosal layer (Fig. 5B). Similarly, GITT was negatively correlated with the number of CD163-positive cells in the colonic muscular layer (Fig 5C).

\section{Discussion}

It has been accepted that antibiotic-related dysbiosis is associated with the development of metabolic disorder and functional gastrointestinal disorders (15). In the present study, we demonstrated that vancomycin treatment clearly promoted a gain of body weight in mice. In addition, we observed that food intake was significantly greater in vancomycin-treated mice, suggesting that vancomycin may promote appetite, thus leading to an increase of body weight gain. Although the types of microbiota that are responsible for induction of obesity remains unclear, our preliminary analyses had shown that the ratio of Lactobacillus was markedly high in vancomycin-treated mice (data not shown), being compatible with previous reports $(11,16)$. Interestingly, it has been reported that Lactobacillus is increased in obese $(17,18)$ and moreover, Lactobacillus species are widely used as growth promoters in the farm industry (19). Together, we are tempting to speculate that the increase of Lactobacillus species associated with vancomycin treatment may be involved at least in part in the obesity phenotype in mice with vancomycin treatment. On the other hand, we clarified that GI motility in mice was suppressed by vancomycin treatment. In this context, since we have recently clarified that intestinal macrophages play a pivotal role in GI motility (20), we investigated the significance of macrophage phenotype alterations in this experimental model.

We first observed the distribution and population of M1 and M2 macrophages in the colonic tissues of mice treated with vancomycin. Although it is impossible to distinguish M1 and M2 macrophage using available markers in vivo 


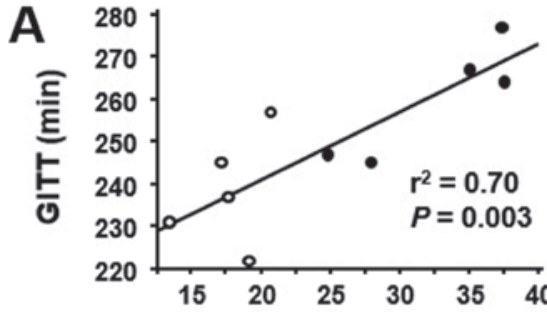

Number of CD80-positive cells In the mucosa of the proximal colon

B

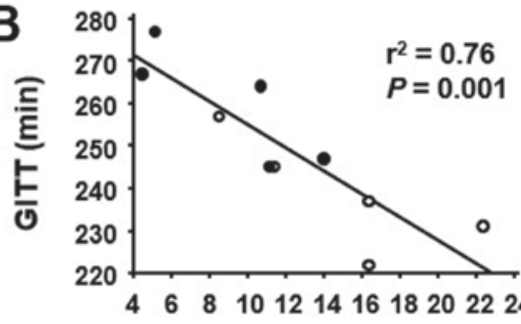

Number of CD163-positive cells In the mucosa of the proximal colon

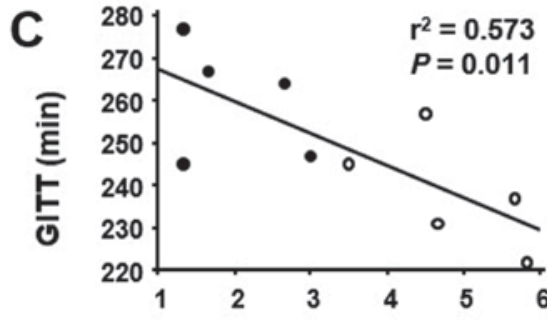

Number of CD163-positive cells In the muscular layer of the proximal colon

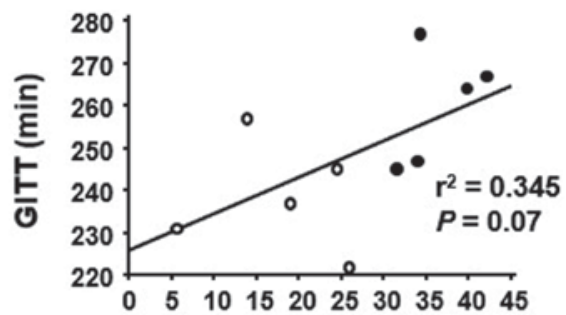

Number of CD80-positive cells

In the mucosa of the distal colon

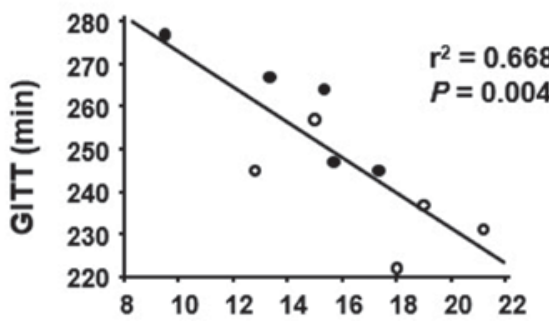

Number of CD163-positive cells In the mucosa of the distal colon

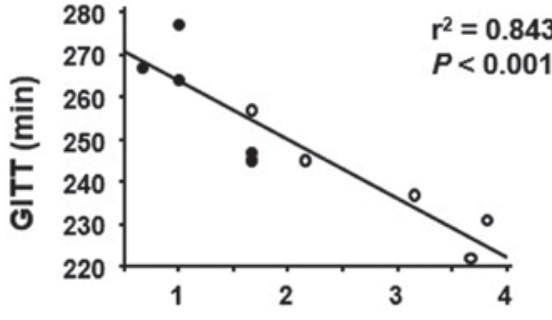

Number of CD163-positive cells In the muscular layer of the distal colon

Figure 5. Correlation between gastrointestinal transit time and CD80 or CD163 expression in the colon of experimental mice. Results from vancomycin-treated mice are presented as black circles $(n=5)$, whereas results from untreated controls are presented as white circles $(n=5)$. P-values were obtained by linear regression analysis comparing GITT with the number of (A) CD80 or (B) CD163-positive macrophages in the mucosal of the colon. (C) Correlation between GITT and the number of CD163-positive macrophages in the muscular layer of the colon. GITT, gastrointestinal transit time.

tissues, we used the CD80 and CD163 that are widely used as a marker for M1 and M2 marker, respectively. Interestingly, we found that M1 macrophages were increased in the colonic mucosa of these mice, and conversely M2 macrophages were decreased in both the colonic mucosa and muscular layer of those mice. These findings suggest that vancomycin-induced dysbiosis greatly affects the macrophage phenotypic profile in colonic tissues. On the whole, macrophage polarization was dominantly shifted toward an M1 phenotype in the colon of this animal model. M1 macrophages are key players in the proinflammatory reaction downstream of IFN- $\gamma$ stimulation (21), and indeed interact with not only other immune cells but also neural, muscle or epithelial cells using proinflmmatory cytokines as mediators (22). On the other hand, M2 macrophages are known to interact with those cells in an anti-inflammatory context (23). Thus, the profile of cytokines expression in vancomycin-treated mice is interesting. The enhancement of proinflammatory IFN- $\gamma$ and IL-12 expression is well compatible with the dominant shift to M1 macrophages in vancomycin-treated mice, and moreover, the decrease of anti-inflammatory IL-4 expression is also consistent with reduction of M2 macrophages in these mice.
Then, what is the influence of the M1-dominant shift of macrophage polarization on the metabolism and GI function? Lumeng et al (24) have reported that obesity induces a phenotypic switch in adipose tissue macrophage polarization, and that thereafter, involvement of M1 macrophages and its associated proinflammatory cytokines play a dominant role in the progression of obesity (25). In particular, the insulin resistance induced by M1 macrophages-associated proinflammatory cytokines are considered as a key mechanism $(24,26,27)$. In this regard, it is interesting that IFN- $\gamma$ expression to activate M1 macrophage is increased and furthermore, M1 macrophage-associated pro-inflammatory cytokine IL-12 was increased in our experimental animals. On the other hand, vancomycin-induced imbalance of macrophage phenotypes may be involved in the alteration of GI motility. Indeed, the present study revealed that an increase of M1 macrophages was significantly correlated with prolongation of the GITT. In fact, previous studies have suggested that M1 macrophages suppress GI motility through the effects of associated proinflammatory cytokines on smooth muscle and the enteric nervous system in an ileus model (28), although this mechanism may not be applicable to our present dysbiosis model. Furthermore, it is noteworthy that M2 
macrophages were suppressed not only in the mucosa but also in the muscular layer in the colon of mice treated with vancomycin. Muller et al (7) and others have reported that muscularis macrophages play a pivotal role in GI motility through cross-talk with enteric nerve cells via cytokines $(29,30)$. Accordingly, the muscularis M2 macrophages in our experimental mice must have played some roles in GI motility. In this context, we have recently demonstrated that $\mathrm{M} 2$ macrophages migrating into the GI muscular layer may be involved in acceleration of GI motility in germ-free mice subjected to commensal bacterial transplantation (20). In contrast, we have shown that reduction of M2 macrophages is correlated with suppression of GI motility in vancomycin-treated mice, which seems reasonable in view of previous work. However, the mechanism by which M2 macrophages regulate GI motility remains to be resolved.

In summary, we have shown that treatment with the antibiotic vancomycin promotes body weight gain and food intake and prolongs the GITT in mice. Moreover, polarization of macrophages shows a shift to M1 phenotype dominance and upregulation of M1 macrophage-associated proinflammatory cytokines in the colon of vancomycin-treated mice. Of note, enhancement of M1 polarization was positively correlated with inhibition of GI motility, whereas suppression of M2 polarization was negatively correlated. These findings suggest that antibiotic treatment may affect body metabolism and GI motility accompanied by alteration of macrophage polarization and the cytokine profile in the colon.

\section{Acknowledgements}

The authors would like to thank Miss Chiyomi Ito and Miss Mayumi Yamada (Hyogo College of Medicine, Nishinomiya, Japan) for their technical assistance.

\section{Funding}

This study was supported in part by Grants-in-aid for Scientific Research (grant no. 17K09363) from the Ministry of Education, Culture, Sports, Science and Technology, Japan.

\section{Availability of data and materials}

The datasets used and/or analyzed during the current study are available from the corresponding author on reasonable request.

\section{Authors' contributions}

YI, HF, XX, YR and HM conceived and designed the experiments. YI, HF, XX and YR performed the experiments. YI, HF, XX, YR, TT, TO and JW analyzed the data. YI, HF, XX, YR, TT and JW contributed reagents/materials/analysis tools. YI, HF, XX and HM were involved in the drafting of the manuscript and revising it critically for important intellectual content. All the authors critically reviewed and approved the manuscript for publication.

\section{Ethics approval and consent to participate}

All experimental protocols were approved by the Animal Use and Care Committee at Hyogo College of Medicine.

\section{Patient consent for publication}

Not applicable.

\section{Competing interests}

The authors declare that they have no competing interests.

\section{References}

1. Tremaroli V and Bäckhed F: Functional interactions between the gut microbiota and host metabolism. Nature 489: 242-249, 2012.

2. Canton J, Neculai D and Grinstein S: Scavenger receptors in homeostasis and immunity. Nat Rev Immunol 13: 621-634, 2013.

3. Jämsen E, Kouri VP, Olkkonen J, Cör A, Goodman SB, Konttinen YT and Pajarinen J: Characterization of macrophage polarizing cytokines in the aseptic loosening of total hip replacements. J Orthop Res 32: 1241-1246, 2014.

4. Mosser DM: The many faces of macrophage activation. J Leukoc Biol 73: 209-212, 2003.

5. Stein M, Keshav S, Harris N and Gordon S: Interleukin 4 potently enhances murine macrophage mannose receptor activity: A marker of alternative immunologic macrophage activation. J Exp Med 176: 287-292, 1992.

6. Novoselov VV, Sazonova MA, Ivanova EA and Orekhov AN: Study of the activated macrophage transcriptome. Exp Mol Pathol 99: 575-580, 2015

7. Muller PA, Koscsó B, Rajani GM, Stevanovic K, Berres ML, Hashimoto D, Mortha A, Leboeuf M, Li XM, Mucida D, et al: Crosstalk between muscularis macrophages and enteric neurons regulates gastrointestinal motility. Cell 158: 300-313, 2014.

8. Theriot CM, Koenigsknecht MJ, Carlson PE Jr, Hatton GE, Nelson AM, Li B, Huffnagle GB, Z Li J and Young VB: Antibiotic-induced shifts in the mouse gut microbiome and metabolome increase susceptibility to Clostridium difficile infection. Nature Commun 5: 3114, 2014.

9. Mahana D, Trent CM, Kurtz ZD, Bokulich NA, Battaglia T, Chung J, Müller CL, Li H, Bonneau RA and Blaser MJ: Antibiotic perturbation of the murine gut microbiome enhances the adiposity, insulin resistance, and liver disease associated with high-fat diet. Genome Med 8: 48, 2016.

10. Cho I, Yamanishi S, Cox L, Methé BA, Zavadil J, Li K, Gao Z, Mahana D, Raju K, Teitler I, et al: Antibiotics in early life alter the murine colonic microbiome and adiposity. Nature 488: 621-626, 2012.

11. Sekirov I, Tam NM, Jogova M, Robertson ML, Li Y, Lupp C and Finlay BB: Antibiotic-induced perturbations of the intestinal microbiota alter host susceptibility to enteric infection. Infect Immun 76: 4726-4736, 2008.

12. Sun C, Fukui H, Hara K, Kitayama Y, Eda H, Yang M, Yamagishi H, Tomita T, Oshima T, Watari J, et al: Expression of Reg family genes in the gastrointestinal tract of mice treated with indomethacin. Am J Physiol Gastrointest Liver Physiol 308: G736-G744, 2015.

13. Kitayama Y, Fukui H, Hara K, Eda H, Kodani M, Yang M, Sun C, Yamagishi $\mathrm{H}$, Tomita T, Oshima $\mathrm{T}$, et al: Role of regenerating gene I in claudin expression and barrier function in the small intestine. Transl Res 173: 92-100, 2016.

14. Yang M, Fukui H, Eda H, Xu X, Kitayama Y, Hara K, Kodani M, Tomita T, Oshima T, Watari J and Miwa H: Involvement of gut microbiota in association between GLP-1/GLP-1 receptor expression and gastrointestinal motility. Am J Physiol Gastrointest Liver Physiol 312: G367-G373, 2017.

15. Mendall MA and Kumar D: Antibiotic use, childhood affluence and irritable bowel syndrome (IBS). Eur J Gastroenterol Hepatol 10: 59-62, 1998.

16. Cheng RY, Li M, Li SS, He M, Yu XH, Shi L and He F: Vancomycin and ceftriaxone can damage intestinal microbiota and affect the development of the intestinal tract and immune system to different degrees in neonatal mice. Pathog Dis 75, 2017.

17. Armougom F, Henry M, Vialettes B, Raccah D and Raoult D: Monitoring bacterial community of human gut microbiota reveals an increase in Lactobacillus in obese patients and Methanogens in anorexic patients. PLoS One 4: e7125, 2009.

18. Million M, Angelakis E, Paul M, Armougom F, Leibovici L and Raoult D: Comparative meta-analysis of the effect of Lactobacillus species on weight gain in humans and animals. Microb Pathog 53: 100-108, 2012. 
19. Khan M, Raoult D, Richet H, Lepidi H and La Scola B Growth-promoting effects of single-dose intragastrically administered probiotics in chickens. Br Poult Sci 48: 732-735, 2007.

20. Yang M, Fukui H, Eda H, Kitayama Y, Hara K, Kodani M, Tomita T, Oshima T, Watari J and Miwa H: Involvement of gut microbiota in the association between gastrointestinal motility and 5-HT expression/M2 macrophage abundance in the gastrointestinal tract. Mol Med Rep 16: 3482-3488, 2017.

21. Hu X, Herrero C, Li WP, Antoniv TT, Falck-Pedersen E, Koch AE, Woods JM, Haines GK and Ivashkiv LB: Sensitization of IFN-gamma Jak-STAT signaling during macrophage activation. Nat Immunol 3: 859-866, 2002.

22. Gordon S: Alternative activation of macrophages. Nat Rev Immunol 3: 23-25, 2003.

23. Zhao A, Urban JF Jr, Anthony RM, Sun R, Stiltz J, van Rooijen N Wynn TA, Gause WC and Shea-Donohue T: Th2 cytokine-induced alterations in intestinal smooth muscle function depend on alternatively activated macrophages. Gastroenterology 135: 217-225, 2008.

24. Lumeng CN, Bodzin JL and Saltiel AR: Obesity induces a phenotypic switch in adipose tissue macrophage polarization. J Clin Invest 117: 175-184, 2007.

25. Lumeng CN, Deyoung SM, Bodzin JL and Saltiel AR: Increased inflammatory properties of adipose tissue macrophages recruited during diet-induced obesity. Diabetes 56: 16-23, 2007.
26. Fujisaka S, Usui I, Bukhari A, Ikutani M, Oya T, Kanatani Y, Tsuneyama K, Nagai Y, Takatsu K, Urakaze M, et al: Regulatory mechanisms for adipose tissue M1 and M2 macrophages in diet-induced obese mice. Diabetes 58: 2574-2582, 2009.

27. Olefsky JM and Glass CK: Macrophages, inflammation, and insulin resistance. Annu Rev Physiol 72: 219-246, 2010

28. Wehner S, Behrendt FF, Lyutenski BN, Lysson M, Bauer AJ, Hirner A and Kalff JC: Inhibition of macrophage function prevents intestinal inflammation and postoperative ileus in rodents. Gut 56: 176-185, 2007.

29. Avetisyan M, Rood JE, Huerta Lopez S, Sengupta R, Wright-Jin E, Dougherty JD, Behrens EM and Heuckeroth RO: Muscularis macrophage development in the absence of an enteric nervous system. Proc Natl Acad Sci USA 115: 4696-4701, 2018.

30. Cipriani G, Gibbons SJ, Kashyap PC and Farrugia G: Intrinsic gastrointestinal macrophages: Their phenotype and role in gastrointestinal motility. Cell Mol Gastroenterol Hepatol 2: 120-130.e1, 2016. 